\title{
Exploiting a Simple Method for the Determination of Manganese in Polyethylene Lined Tubing for Petroleum and Natural Gas Industries
}

\author{
Xiaodong Shao ${ }^{1,2 *}$, and Xin $\mathrm{Shi}^{3}$ \\ ${ }^{1}$ State Key Laboratory for Performance and Structure Safety of Petroleum Tubular Goods and \\ Equipment Materials, No. 89 Jinyeer Road, Xi'an 710077, China \\ ${ }^{2}$ CNPC Tubular Goods Research Institute, No. 89 Jinyeer Road, Xi'an 710077, China \\ ${ }^{3}$ Engineering Institute of Technology, Sinopec Northwest Oilfield Branch, Urumqi 830011, China
}

\begin{abstract}
The polyethylene lined tubing is the key to enabling the industry to meet some of the energy security challenges that nations face today. It is well known that manganese is an important element in polyethylene lined tubing. In this paper, a simple spectrophotometric method was described for the determination of manganese in polyethylene lined tubing. The method was based on the oxidation-reduction reaction between ammonium persulfate and manganese(II) producing manganese(VII) in the presence of silver nitrate as a catalyst. The characteristic wavelength of maximum absorption of manganese(VII) was obtained locating at $530 \mathrm{~nm}$. Under the optimum reaction conditions the absorption value was proportional to the concentration of manganese in the range of $0.2 \% \sim 1.9 \%\left(\mathrm{R}^{2}=0.9997\right)$, and the relative standard deviation was less than $3.0 \%(n=5)$. The proposed method was applied successfully to determine manganese in polyethylene lined tubing real samples.
\end{abstract}

\section{Introduction}

In the west of China, the area has rich petroleum resource, which has become an important exploitation target now. The condition of gas and oil wells in the west of China has complex characteristics such as deep, high temperature, high pressure, and serious corrosive that only corrosionresistant tubing can be applied to explore. One key feature there is obvious existence of corrosive
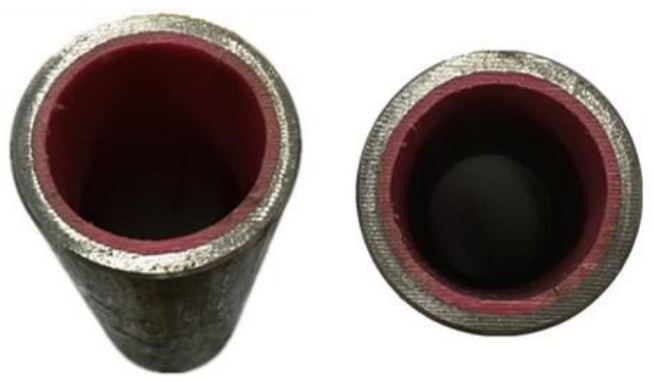

Fig. 1. Polyethylene lined tubing samples

*Corresponding author: shaoxd@163.com 
hydrogen sulfide medium. The sulfide stress corrosion failure frequently occurs due to absence of applicable technical specification which has led to huge economic damage. Statistical data showed that the average loss is 100 thousand dollar for the tubing failure. In some areas no suitable tubing can be supplied due to high hydrogen sulfide content. So there is obvious safety risk when tubing in these gas fields. Specification for tubing used in these regions was very necessary and urgent. The polyethylene lined tubing (Fig. 1) has excellent resistance to corrosion. These composite pipes are key to enabling the industry to meet some of the energy security challenges that nations face today. Thus, polyethylene lined tubing will be widely used in the exploitation of acid gas and oil wells. The content of chemical elements in polyethylene lined tubing directly influences the properties of materials. In order to control the quality of polyethylene lined tubing products, the contents of trace impurities and alloy elements in polyethylene lined tubing should be determined. In making processes, the content of alloyed elements such as manganese, nickel, chromium, molybdenum, vanadium, and so on, is required to be strictly controlled because it fundamentally determines the performance of polyethylene lined tubing.

It is well known that manganese is an important element in polyethylene lined tubing. Therefore, a rapid and precise analytical method for the determination of manganese is essentially required for the production control of polyethylene lined tubing. Manganese is valuable alloying agents and coexists in a number of industrially important alloys and steels. Owing to their increasing industrial applications of manganese, the determination of trace amounts of manganese is of great interest. Therefore, it was important from the analytical point of view to develop simple, sensitive and reliable methods for the determination of manganese in various complex matrixes. Numerous analytical methods were employed for the determination of manganese in different types of matrices including atomic emission spectrophotometry [1,2], atomic absorption spectrometry [3], laser induced breakdown spectroscopy [4], atomic fluorescence spectroscopy [5], neutron activation analysis [6], electroanalytical method [7] and spectrophotometry [8]. Spectrophotometric methods occupy special position due to their simplicity, less expensive instrumentation and high sensitivity. Similarly, spectrophotometric methods also play an important role in manganese determination. The literatures shows that aromatic amines, azo dyes, oximes, porphyrins and other reagents have been used for the colorimetric determination of manganese. The most significant reagents are permanganate, formaldoxime and 1-(2pyridylazonaphthol), which is far more sensitive than the other two reagents. This present method was based on the oxidation-reduction reaction between ammonium persulfate and manganese(II). Employing ammonium persulfate as an oxidant, manganese(II) was oxidized to manganese(VII) in the presence of silver nitrate as a catalyst at the room temperature. The characteristic wavelength of maximum absorption was measured at 530 $\mathrm{nm}$. Under the optimum reaction conditions the absorption value $(A)$ was proportional to the concentration of manganese in the range of $0.2 \% \sim 1.9 \%$ (mass fraction), and the relative standard deviation (RSD) was less than $3.0 \%(n=5)$. The proposed method was free from the interference from a large number of analytical important elements and has been applied satisfactorily to the determination of manganese in polyethylene lined tubing samples with the improved accuracy and precision. Results were in agreement with those attained by inductively coupled plasma atomic emission spectrophotometry at the $95 \%$ confidence level. The satisfactory performance in the determination of manganese in polyethylene lined tubing demonstrated that the method was practical and suitable not only for quality control analysis but also for product analysis, confirming the promise for polyethylene lined tubing research. 


\section{Experiment}

\subsection{Apparatus}

A UV-visible spectrophotometer (Model TU-1901, Beijing Persee General Instrument Co. Ltd.) with 1.0-cm quartz cells was employed for all absorbance measurements.

\subsection{General procedures}

Weigh accurately $0.1000 \mathrm{~g}$ of drilled low alloy steel samples and dissolve the samples in 20 $\mathrm{mL}$ mixture acid solution of sulphuric acid $15 \%(\mathrm{~V} / \mathrm{V})$ and phosphoric acid $15 \%(\mathrm{~V} / \mathrm{V})$ in a $150-\mathrm{mL}$ taper bottle. Continue the treatment with mixture acid till the sample goes into solution and heat to fumes of oxides of nitrogen, sulphuric acid and evaporate almost to dryness. Cool the solution, add $50 \mathrm{~mL}$ deionised water to dissolve some materials. Then, add $5 \mathrm{~mL}$ of $5.0 \mathrm{mg} \cdot \mathrm{mL}^{-1}$ solution of silver nitrate, $10 \mathrm{~mL}$ of $200.0 \mathrm{mg} \cdot \mathrm{mL}^{-1}$ solution of ammonium persulfate in the taper bottle to oxidate manganese(II) to manganese(VII) oxidation states. Boil the solution to no bubble and cool the solution at room temperature. Finally, dilute the solution in a $100-\mathrm{mL}$ volumetric flask, followed make up to the mark with deionised water, mix well and let stand for $10 \mathrm{~min}$. Then, transfer the above mulberry stock solution in $1.0-\mathrm{cm}$ quartz cells. Add $5.0 \mathrm{mg} \cdot \mathrm{mL}^{-1}$ solution of sodium nitrite to the remaining solution reduce manganese(VII) to the lower oxidation states, this transparent solution was employed as the blank solution in subsequent experiments. Measure the absorbance at $530 \mathrm{~nm}$ using a UV-visible spectrophotometer with 1.0-cm quartz cells against a reagent blank prepared similarly. Plot the amount of manganese in the sample solution against absorbance to obtain the calibration graph.

\section{Results and discussion}

\subsection{Spectral characteristics}

The absorption spectra of the reagents blank, manganese(VII) against the reagent blank were tested from $400 \mathrm{~nm}$ to $700 \mathrm{~nm}$. The manganese(VII) yields the characteristic wavelength of maximum absorption at $530 \mathrm{~nm}$ wavelength. However, the absorption spectrum of the reagents blank under similar conditions shown that it does not absorb at $530 \mathrm{~nm}$. And the experimentation also demonstrated that manganese(VII) increases the absorbance value considerably, resulting in increased sensitivity of the method. Therefore, measure the absorbance of manganese(VII) at $530 \mathrm{~nm}$.

\subsection{Select dissolved acid for samples}

The effect of the different acids for dissolve the low alloy steel samples was examined. It was found that a solution mixed with $15 \%(\mathrm{~V} / \mathrm{V})$ sulphuric acid and $15 \%(\mathrm{~V} / \mathrm{V})$ phosphoric acid has a good effect for dissolve the low alloy steel samples. Thus, an acid solution mixed with $15 \%(\mathrm{~V} / \mathrm{V})$ sulphuric acid and $15 \%(\mathrm{~V} / \mathrm{V})$ phosphoric acid was recommended for dissolve the low alloy steel samples in subsequent studies. 


\subsection{Influence of the amounts of oxidant}

Ammonium persulfate was selected as the oxidant in the proposed method. The effect of the oxidant was examined by measuring the absorbance of solution containing certain amounts of manganese(II) and variable amounts of ammonium persulfate. It was found that $10 \mathrm{~mL}$ of $200.0 \mathrm{mg} \cdot \mathrm{mL}^{-1}$ solution of ammonium persulfate sufficed to oxidate the amounts of manganese(II) taken, with higher concentrations the absorbance was essentially constant. Ten millilitres of $200.0 \mathrm{mg} \cdot \mathrm{mL}^{-1}$ ammonium persulfate solution were recommended as a suitable amount of oxidant.

\subsection{Influence of the amounts of silver nitrate}

The oxidation-reduction reaction between manganese(II) and ammonium persulfate without a catalyst was slowly and not fully. Selected the silver nitrate as a catalyst for the oxidationreduction reaction between manganese(II) and ammonium persulfate. It has been further observed that the oxidation-reduction reaction between manganese(II) and ammonium persulfate occurs immediately even at room temperature if silver nitrate was used. Therefore, $5 \mathrm{~mL}$ of $5.0 \mathrm{mg} \cdot \mathrm{mL}^{-1}$ solution of silver nitrate was employed in subsequent experiments.

\subsection{Influence of the reductant}

Sodium nitrite was selected as the reductant in the proposed method. $5.0 \mathrm{mg} \cdot \mathrm{mL}^{-1}$ solution of sodium nitrite reduce manganese(VII) to the lower oxidation states manganese(II). The transparent solution was employed as the blank solution in subsequent experiments.

\subsection{Sequence of addition of reagents}

From experiments in which sulphuric acid, phosphoric acid, ammonium persulfate and silver nitrate were added in all possible orders. It was found that there was no appreciable change in the colour of the solution and the variations in the absorbance values. These phenomenons demonstrate that the oxidation-reduction reaction between manganese(II) and ammonium persulfate was independent of the order of addition.

\subsection{Preparation of the calibration graph}

Under the optimal conditions, a series of standard solutions of manganese were prepared by the different steel certified reference materials in a $100-\mathrm{mL}$ volumetric flask. Add $5 \mathrm{~mL}$ of $5.0 \mathrm{mg} \cdot \mathrm{mL}^{-1}$ solution of silver nitrate, $10 \mathrm{~mL}$ of $200.0 \mathrm{mg} \cdot \mathrm{mL}^{-1}$ solution of ammonium persulfate followed make up to the mark with deionised water, mix well and let stand for 10 min. Measure the absorbance at $530 \mathrm{~nm}$ using a UV-visible spectrophotometer with $1.0-\mathrm{cm}$ quartz cells against a reagent blank. The absorbance of the solution was proportional to the concentration of manganese in the range of $0.2 \% \sim 1.9 \%$ (mass fraction). Plot the amount of manganese in the sample solution against absorbance to obtain the calibration graph. The linear regression equation for manganese was $\mathrm{A}=0.41837 \mathrm{C}-0.01073, \mathrm{R}^{2}=0.9997$, and the relative standard deviation (RSD) was less than $3.0 \%(n=5)$. 


\subsection{Interference studies}

The extent of interference by diverse ions was determined by measuring the absorbance of solutions containing manganese and various amounts of diverse ions. The criterion for interference was an absorbance value varying by more than $+2 \%$ from the expected value of manganese alone. The results show that a large excess of cations and anions which are usually associated in the determination of manganese do not interfere. The colorless metal ions do not interfere. The tolerable amounts of foreign species with respect to manganese for interference at $+2 \%$ level were over $200 \sim 300$ for the colored metal ions. When the colored metal ions contents of not more than 200 300 times manganese, it can be in some color solution adding a few drops of EDTA solution or sodium nitrite solution to fade the color, this solution as the measured reference solution to eliminate the interference. There was not obvious interference ions exist in polyethylene lined tubing samples.

\section{Applications}

\subsection{Determination of manganese in polyethylene lined tubing samples}

The recommended procedure has been applied satisfactorily to the determination of manganese in polyethylene lined tubing samples. Measure the absorbance at $530 \mathrm{~nm}$ using a UV-visible spectrophotometer with $1.0-\mathrm{cm}$ quartz cells against a reagent blank. The results were summarized in Table 1 with the good accuracy and precision. To verify the results obtained by the proposed method, inductively coupled plasma atomic emission spectrophotometry method was applied to determine the samples according to the literature and the results obtained by the proposed method agreed well with that from the reference method.

Table 1. Results of determination of manganese in polyethylene lined tubing samples ${ }^{\mathrm{a}}$

\begin{tabular}{|c|c|c|c|c|c|}
\hline \multirow{2}{*}{$\begin{array}{l}\text { Sample } \\
\text { No. }\end{array}$} & \multirow[b]{2}{*}{ Size (mm) } & \multirow[b]{2}{*}{$A$} & \multirow[b]{2}{*}{ RSD (\%) } & \multicolumn{2}{|c|}{ Content $(\omega, \%)$} \\
\hline & & & & $\begin{array}{c}\text { By the proposed } \\
\text { method }\end{array}$ & By AES \\
\hline 1 & $\Phi 73$ & 0.2570 & 2.51 & 0.64 & 0.65 \\
\hline 2 & $\Phi 73$ & 0.2545 & 1.87 & 0.63 & 0.63 \\
\hline 3 & $\Phi 73$ & 0.5289 & 0.96 & 1.29 & 1.32 \\
\hline 4 & $\Phi 73$ & 0.5373 & 1.09 & 1.31 & 1.30 \\
\hline 5 & $\Phi 73$ & 0.5457 & 2.16 & 1.33 & 1.33 \\
\hline 6 & $\Phi 73$ & 0.5444 & 1.12 & 1.33 & 1.35 \\
\hline 7 & $\Phi 73$ & 0.5708 & 0.66 & 1.39 & 1.41 \\
\hline 8 & $\Phi 73$ & 0.5624 & 0.79 & 1.37 & 1.35 \\
\hline 9 & $\Phi 89$ & 0.1943 & 2.77 & 0.49 & 0.51 \\
\hline 10 & $\Phi 89$ & 0.2068 & 2.25 & 0.52 & 0.50 \\
\hline 11 & $\Phi 89$ & 0.5917 & 1.53 & 1.44 & 1.46 \\
\hline 12 & $\Phi 89$ & 0.6043 & 1.24 & 1.47 & 1.48 \\
\hline 13 & $\Phi 89$ & 0.4829 & 0.92 & 1.18 & 1.16 \\
\hline 14 & $\Phi 89$ & 0.4913 & 0.30 & 1.20 & 1.20 \\
\hline 15 & $\Phi 89$ & 0.2236 & 1.59 & 0.56 & 0.56 \\
\hline 16 & $\Phi 89$ & 0.2213 & 2.00 & 0.55 & 0.57 \\
\hline
\end{tabular}

Notes: ${ }^{\text {a }}$ The average of 5 determinations. 


\section{Conclusions}

Based on the oxidation-reduction reaction between manganese(II) and ammonium persulfate, a simple and accurate spectrophotometric method for determination of manganese in polyethylene lined tubing was proposed. The purple-red colored manganese(VII) solution exhibits absorption maximum at $530 \mathrm{~nm}$. The proposed method was free from the interference from a large number of analytical important elements and has been applied satisfactorily to the determination of manganese in polyethylene lined tubing samples. Compared with other methods for the determination of manganese, this method offers advantages of simplicity, less expensive instrumentation and high efficient. The satisfactory performance in the determination of manganese in polyethylene lined tubing demonstrated that the method was practical and suitable not only for quality control analysis but also for product analysis, confirming the promise for polyethylene lined tubing research.

The authors gratefully acknowledge the State Key Laboratory for Performance and Structure Safety of Petroleum Tubular Goods and Equipment Materials, and CNPC Tubular Goods Research Institute.

\section{References}

1. W. Li, P. Simmons, D. Shrader, T.J. Herrman, S.Y. Dai, Talanta 112, 43 (2013)

2. N. Ozbek, S. Akman, Food Chemistry 200, 245 (2016)

3. S.M.A. Azeem, S.M.M. Attaf, M.F. El-Shahat, Reactive and Functional Polymers 73, $182(2013)$

4. M.R. Pourjavid, A.A. Sehat, Microchemical Journal 129, 259 (2016)

5. S. Feng, D. Yuan, T. Zhou, Analytica Chimica Acta 963, 53 (2017)

6. K. Tomura, H. Tomuro, Journal of Radioanalytical and Nuclear Chemistry 242, 147 (1999)

7. R. Piech, B. Baśa, W.W. Kubiaka, Journal of Electroanalytical Chemistry 621, 43 (2008)

8. S. Feng, Y. Huang, T. Zhou, Continental Shelf Research 92, 37 (2015) 